OPEN ACCESS

Edited by:

Walter Gottlieb Land, University of Strasbourg, France

Reviewed by: Anne Cooke, University of Cambridge, UK

Dominic De Nardo,

Walter and Eliza Hall Institute of Medical Research, Australia

${ }^{*}$ Correspondence:

Carolina Pellegrini carolina.pellegrini87@gmail.com

Specialty section: This article was submitted to Inflammation, a section of the journa Frontiers in Immunology

Received: 20 October 2016 Accepted: 09 January 2017 Published: 25 January 2017

Citation:

Pellegrini C, Antonioli L, LopezCastejon G, Blandizzi $C$ and Fornai $M$ (2017) Canonical and Non-Canonical Activation of NLRP3 Inflammasome at the Crossroad between Immune Tolerance and Intestinal Inflammation.

Front. Immunol. 8:36.

doi: 10.3389/fimmu.2017.00036

\section{Canonical and Non-Canonical Activation of NLRP3 Inflammasome at the Crossroad between Immune Tolerance and Intestinal Inflammation}

\author{
Carolina Pellegrini, ${ }^{1 *}$, Luca Antonioli', Gloria Lopez-Castejon², Corrado Blandizzi' and \\ Matteo Fornai ${ }^{1}$ \\ ${ }^{1}$ Department of Clinical and Experimental Medicine, University of Pisa, Pisa, Italy, ${ }^{2}$ Manchester Collaborative Centre for \\ Inflammation Research, University of Manchester, Manchester, UK
}

Several lines of evidence point out the relevance of nucleotide-binding oligomerization domain leucine rich repeat and pyrin domain-containing protein 3 (NLRP3) inflammasome as a pivotal player in regulating the integrity of intestinal homeostasis and shaping innate immune responses during bowel inflammation. Intensive research efforts are being made to achieve an integrated view about the protective/detrimental role of canonical and non-canonical NLRP3 inflammasome activation in the maintenance of intestinal microenvironment integrity. Evidence is also emerging that the pharmacological modulation of NLRP3 inflammasome could represent a promising molecular target for the therapeutic management of inflammatory immune-mediated gut diseases. The present review has been intended to provide a critical appraisal of the available knowledge about the role of canonical and non-canonical NLRP3 inflammasome activation in the dynamic interplay between microbiota, intestinal epithelium, and innate immune system, taken together as a whole integrated network regulating the maintenance/breakdown of intestinal homeostasis. Moreover, special attention has been paid to the pharmacological modulation of NLRP3 inflammasome, emphasizing the concept that this multiprotein complex could represent a suitable target for the management of inflammatory bowel diseases.

Keywords: canonical, non-canonical, NLRP3, bowel inflammation, intestinal homeostasis, immune system, enteric microbiota

\section{INTRODUCTION}

A growing body of evidence highlights the relevance of the nucleotide-binding oligomerization domain leucine rich repeat and pyrin domain-containing protein 3 (NLRP3) inflammasome in the pathophysiology of several autoinflammatory syndromes [i.e., cryopyrin-associated autoinflammatory syndromes (CAPS), Schnitzler's syndrome], as well as metabolic and/or inflammatory disorders (i.e., obesity, atherosclerosis, type 2 diabetes, gout, and intestinal inflammation) (1-3). In the setting of intestinal microenvironment, NLRP3 inflammasome plays a pivotal role both in regulating the integrity of intestinal homeostasis and in shaping innate immune responses during bowel inflammation (3). In particular, the NLRP3 inflammasome, through the adaptor protein apoptosis-associated 
speck-like protein (ASC), recruits and activates caspase-1 leading to processing and release of IL-18 and IL-1 $\beta$. These are two key cytokines involved both in the control of immune tolerance and support to immune and tissue events occurring in the presence of inflammation (4). This pathway is currently designated as "canonical NLRP3 inflammasome activation." In addition, a "noncanonical NLRP3 inflammasome activation," which depends on caspase-11 in mice (human orthologs are caspase 4 and caspase $5)$, has been described to be pivotal in the maintenance of intestinal immune homeostasis (5).

Several lines of preclinical evidence have unraveled a dual role of NLRP3 inflammasome in the pathogenesis of bowel inflammation (6). In particular, some studies showed a regulatory and reparative role of NLRP3 in the maintenance of immune tolerance and epithelial barrier integrity $(7,8)$. Conversely, others reported that the overactivation of NLRP3 inflammasome during intestinal inflammation is associated with a breakdown of intestinal immune balance, with consequent detrimental effects to the host (9). In this context, research efforts are currently being focused on a better understanding of the role of canonical and non-canonical NLRP3 inflammasome in the pathophysiology of intestinal inflammation.

Based on the above background, the present review has been intended to provide an integrated and critical appraisal of the available knowledge about the protective or detrimental role of canonical and non-canonical NLRP3 inflammasome activation in the maintenance of intestinal homeostasis as well as in sustaining the pathophysiological events underlying bowel inflammation. Special attention has been paid to point out how NLRP3 inflammasome influences the dynamic interplay between microbiota, intestinal epithelium, and innate immune system, as well as how the pharmacological modulation of this enzymatic complex could represent a suitable strategy in the management of inflammatory bowel diseases (IBDs).

\section{MECHANISMS OF CANONICAL AND NON-CANONICAL NLRP3 INFLAMMASOME ACTIVATION}

Canonical NLRP3 inflammasome activation requires two parallel and independent steps: transcription and oligomerization (Figure 1) (10). The first step is regulated by innate immune signaling, mediated primarily by toll-like receptor (TLR)-adaptor molecules myeloid differentiation primary response 88 (MyD88) and/or cytokine receptors, such as the tumor necrosis factor receptor, which, in turn, activate pro-IL-1 $\beta$ and NLRP3 transcription via nuclear factor- $\kappa \mathrm{B}(\mathrm{NF}-\kappa \mathrm{B})$ activation (11). The second step results in NLRP3 inflammasome oligomerization, leading to caspase- 1 activation and, in turn, IL- $1 \beta$ and IL-18 processing and release (12). Various stimuli associated with infections, including an increase in extracellular adenosine triphosphate (ATP), extracellular osmolarity or $\mathrm{pH}$ alterations, $\beta$-amyloid fibers and degradation of extracellular matrix components, increase in potassium efflux, reactive oxygen species (ROS), cathepsin activation, and deubiquitination, can promote NLRP3 inflammasome oligomerization and activation by initiating assembly of a multiprotein complex consisting of NLRP3, the adaptor protein ASC, and pro-caspase-1. The recruitment of ASC is pivotal for the activation of pro-caspase-1 into its cleaved form (13-18). Caspase- 1 activation promotes also, independently from IL-1 $\beta$ maturation, pyroptosis, a key defense mechanism against microbial infections, which blocks the replication of intracellular pathogens via cytoplasmic swelling and promotes phagocytosis of surviving bacteria (19-21). In particular, recent evidence has shown that caspase-1 cleaves the linker between the amino-terminal gasdermin- $\mathrm{N}$ and carboxy-terminal gasdermin- $\mathrm{C}$ domains in gasdermin $\mathrm{D}$, an acid cytoplasmic protein, which plays a critical role in the process of pyroptosis $(22,23)$. Pyroptosis then promotes the release of additional cytosolic proteins, such as high mobility group box 1 (HMGB1) alarmin, a pro-inflammatory mediator significantly involved in the pathogenesis of several inflammatory chronic diseases (Figure 1) (24-26).

Besides canonical NLRP3 inflammasome activation, a noncanonical caspase-11-dependent NLRP3 activation has been characterized (Figure 1) (5). In particular, Gram-negative bacteria (i.e., Citrobacter rodentium, Escherichia coli, Legionella pneumophila, Salmonella typhimurium, and Vibrio cholerae) activate the TLR4-MyD88 and toll/IL-1 receptor homologydomain-containing adapter-inducing interferon- $\beta$ (TRIF) pathways, with a consequent nuclear translocation of NF- $\kappa \mathrm{B}$, which in turn promotes the transcription of IL-1 $\beta$, IL-18, and NLRP3 as well as interferon regulatory factor (IRF)-3 and IRF7 genes $(27,28)$. Subsequently, the IRF3-IRF7 complex elicits the expression of interferon (IFN) $-\alpha / \beta$, which binds the IFN- $\alpha / \beta$ receptor 1 (IFNAR)/IFNAR2 receptor leading to activation of the JAK/ STAT pathway and consequent transcription of caspase-11 gene $(19,29-31)$. In addition, binding of lipopolysaccharide (LPS) to caspase-11 and/or as-yet-unidentified scaffold proteins or receptors induced by Gram-negative bacteria, escaping phagosomes, have been shown to activate the effector functions of caspase-11 $(32,33)$. In particular, once activated, caspase-11 induces pyroptosis through cleavage of gasdermin, as well as HMGB1 and IL- $1 \alpha$ release, and promotes IL- $1 \beta$ processing and release through activation of the NLRP3-ASC-caspase-1 pathway (Figure 1) (22, $23,32)$.

These different NLRP3 activation processes occur independently. However, caspase-11 enhances the canonical caspase-1 processing and IL-1 $\beta /$ IL-18 production in the presence of specific stimuli (e.g., cholerae toxin or E. coli) $(5,22)$. In this setting, further in vitro experiments on cultured cells should be implemented to clarify the molecular mechanisms underlying the interplay between caspase- 1 and -11 in promoting the canonical and/or non-canonical NLRP3 inflammasome activation.

\section{NLRP3 INFLAMMASOME IN THE PATHOPHYSIOLOGY OF BOWEL INFLAMMATION}

A dynamic interplay between enteric microbiota, intestinal epithelium, and mucosal immune system contributes to the maintenance of intestinal homeostasis (34). Indeed, dysbiosis, alterations of intestinal epithelial barrier and uncontrolled 


\section{Canonical NLRP3 inflammasome activation}

Step 1: Trascription

Microbes

(i.e.LPS)
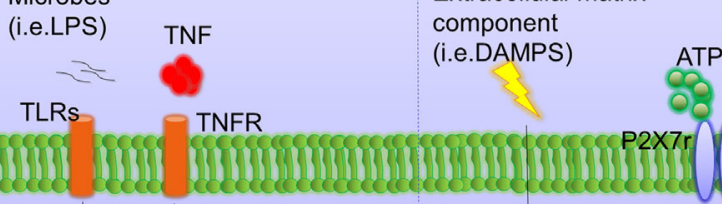

Step 2: Oligomerization

Potassium $\mathrm{K}^{+}$

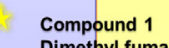

Step 2: caspase-11 activation and NLRP3

oligomerization

Unidentified scaffold proteins or receptors
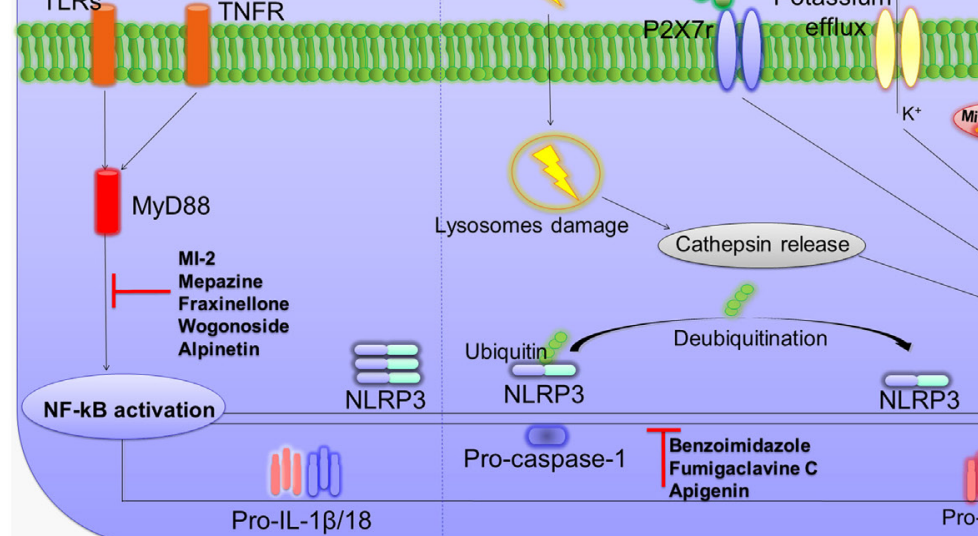

Ros

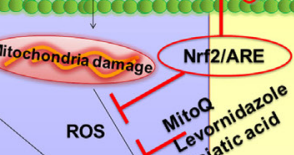

NLRP3 inflammasome activation

Mepazine
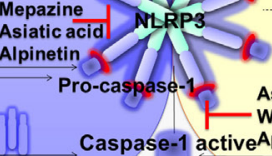

Asiatic acid

IL-1 $\beta$ IL-18

pigenin
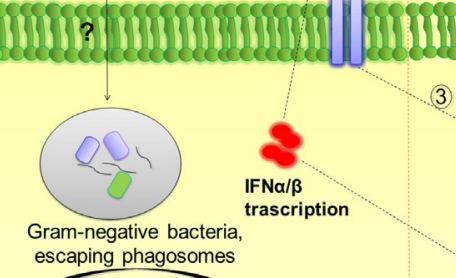

escaping phagosomes
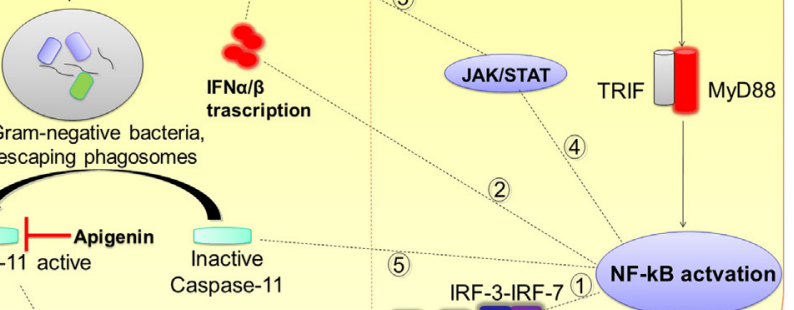

Gram-negative bacteria (i.e. Citrobacter rodentium, Escherichia colli, cholerae)
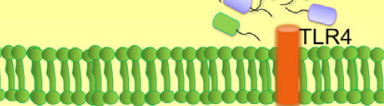

(2)

(4)

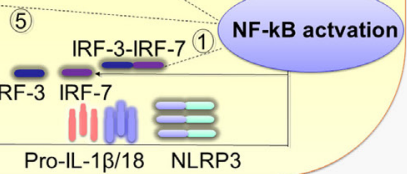

FIGURE 1 Mechanisms of canonical and non-canonical NLRP3 inflammasome activation. Diagram showing the canonical and non-canonical NLRP3 inflammasome activation, and representation of the molecular mechanisms through which several compounds inhibit NLRP3 activation and counteract intestinal inflammation. Left panel: first step of canonical NLRP3 inflammasome activation by TLRs-MyD88 and/or TNFR, which activate pro-IL-1 $\beta$ and NLRP3 transcription via NF-kB activation. The second step results in NLRP3 inflammasome oligomerization, leading to caspase-1 activation as well as IL-1 $\beta$ and IL-18 release. Extracellular ATP, degradation of extracellular matrix components, increase in potassium efflux, ROS, cathepsin activation, and deubiquitination promote NLRP3 inflammasome oligomerization and activation.

Caspase-1 activation promotes also pyroptosis and HMGB1 release. Right panel: first step of non-canonical NLRP3 inflammasome activation. Gram-negative bacteria (i.e., Citrobacter rodentium, Escherichia coli, and Vibrio cholerae) activate the TLR4-MyD88 and TRIF pathways, with consequent nuclear translocation of NF-kB, which promotes the transcription of IL-1 1 , IL-18, and NLRP3 as well as IRF-3 and IRF7 genes. The IRF3-IRF7 complex (1) elicits the expression of IFN- $\alpha / \beta$ (2) that binds the IFNAR1/IFNAR2 receptor (3), leading to activation of the JAK/STAT pathway (4) and transcription of caspase-11 gene (5). In the second step, unidentified scaffold proteins or receptors induced by Gram-negative bacteria cleave and activate caspase-11, which induces pyroptosis as well as HMGB1 and IL-1 $\alpha$ release, and promotes the activation of NLRP3-ASC-caspase-1 pathway. Abbreviations: NLRP3, nucleotide-binding oligomerization domain, leucine rich repeat and pyrin domain-containing protein 3; TLRs, toll-like receptors; MyD88, adaptor molecules myeloid differentiation primary response 88; TNFR, tumor necrosis factor receptor; NF-kB, nuclear factor-kB; ATP, adenosine triphosphate, ROS, reactive oxygen species; HMGB1, high mobility group box 1; TRIF, toll/LL-1 receptor homology (TIR)-domain-containing adapter-inducing interferon- $\beta$; IRF, interferon regulatory factor; IFN, interferon; IFNAR, interferon- $\alpha / \beta$ receptor; IL, interleukin; P2X, purinergic receptor 7; JAK/ STAT, janus kinase/signal transducers and activators of transcription; NRF2/ARE, nuclear factor (erythroid-derived 2)-like 2/antioxidant response element. 
immune responses to pathogenic stimuli represent the main factors implicated in the pathogenesis of bowel inflammation. IBDs, including Crohn's disease and ulcerative colitis, comprise chronic and relapsing inflammatory disorders that affect the gastrointestinal tract (35). In this context, NLRP3 inflammasome has been found to act as a key player both in the maintenance and breakdown of intestinal immune tolerance. Indeed, through the regulation of intestinal epithelial and immune innate cells (monocytes, macrophages and dendritic cells), it contributes to maintaining intestinal homeostasis, while sustaining also the pathophysiological events underlying bowel inflammation (36). However, despite more than a decade has passed since the discovery of inflammasomes, the role of NLRP3 inflammasome in the intestinal homeostasis as well as in the pathophysiology of bowel inflammation remains multifaceted and controversial (6). A number of preclinical investigations have attempted to unravel the role played by NLRP3 inflammasome in this setting. Accordingly, current data on the involvement of canonical and non-canonical NLRP3 pathways in the pathophysiology of bowel inflammation are addressed in the following section.

\section{Canonical NLRP3 Inflammasome Activation}

In an attempt of understanding the role of canonical NLRP3 inflammasome in the pathophysiology of bowel inflammation, several efforts have been made to implement research on the effects of NLRP3 gene deletion and its components on immune and non-immune cell activity, as well as on pathophysiological events downstream its activation in preclinical models of colitis (see Table 1). Two initial reports showed that NLRP3 plays a key role in the regulation of intestinal homeostasis, maintaining the epithelial barrier integrity and reducing mortality during experimental colitis $(7,37)$. In particular, $\mathrm{Nlrp3}^{-/-}, \mathrm{Asc}^{-/-}$, and caspase $-1^{-1-}$ mice were found to be more susceptible to colitis induced by dextran sodium sulfate (DSS) and 2,4,6-trinitrobenzenesulfonic acid (TNBS), both characterized by body weight loss, diarrhea, rectal bleeding, and mortality, suggesting a protective role of NLRP3 inflammasome in the digestive tract. Such a favorable action was ascribed to the ability of NLRP3 of inducing IL-18 release, a crucial mediator in the repair of colonic mucosal barrier that, through binding IL-18 receptors on intestinal epithelial cells, exerts a restorative effect on the enteric epithelium (Figure 2). In addition, $N l r p 3^{-1-}$ mice showed an elevation of nitric oxide (NO) levels, likely resulting from an increase in inducible NO synthase (iNOS) activity, and a decrease in the anti-inflammatory IL-10 cytokine and protective growth factor TGF- $\beta$ expression, thus suggesting the ability of NLRP3 to regulate the production of pro- and anti-inflammatory mediators in the presence of bowel inflammation (7). However, the molecular mechanisms underlying NLRP3 inflammasome-dependent regulation of these inflammatory factors remain to be determined. In the same paper, the authors showed also that NLRP3 activation modulated the activity and trafficking of neutrophils as well as leukocyte recruitment. In particular, NLRP3-deficient neutrophils showed a pattern of altered migration, attenuated chemotactic responses, and enhanced spontaneous apoptosis (7). These findings support the view that NLRP3 inflammasome acts in a reparative key, regulating neutrophil and leukocyte phagocytic activity.

Consistent with the above results, Zaki et al. observed that the induction of colitis in Nlrp $3^{-/}$mice was associated with a disruption of intestinal epithelial barrier and an increase in mucosal permeability as compared to DSS wild-type (WT) mice, with consequent bacterial translocation into the mucosa and systemic dissemination (37). Such detrimental effect on the intestinal epithelial barrier, besides a decrease in the inflammasome-dependent IL-18 cytokine release, resulted from the ability of NLRP3 to regulate crypt bactericidal capacity and the expression of colonic $\beta$-defensin, an antimicrobial peptide released by macrophages implicated in the resistance of epithelial surfaces to microbial colonization (Figure 2) (7). These findings highlight the relevance of NLRP3 in modulating the interplay between intestinal epithelium and innate immune cells, suggesting a

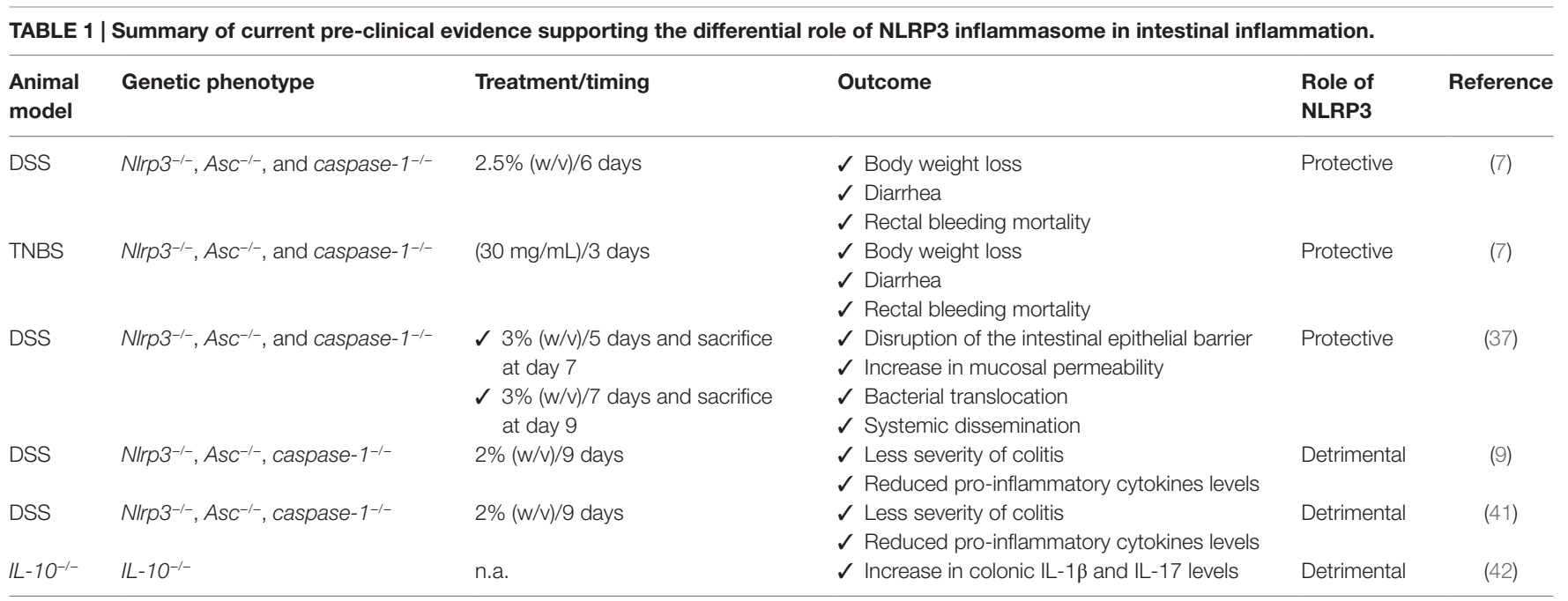

DSS, dextran sodium sulfate; TNBS, 2,4,6-trinitrobenzenesulfonic acid; w/V, weight/volume; IL-1ß, interleukin-1beta; IL-17, interleukin-17. 
Intestinal homeostasis

Canonical NLRP3 inflammasome

Non-canonical NLRP3 inflammasome

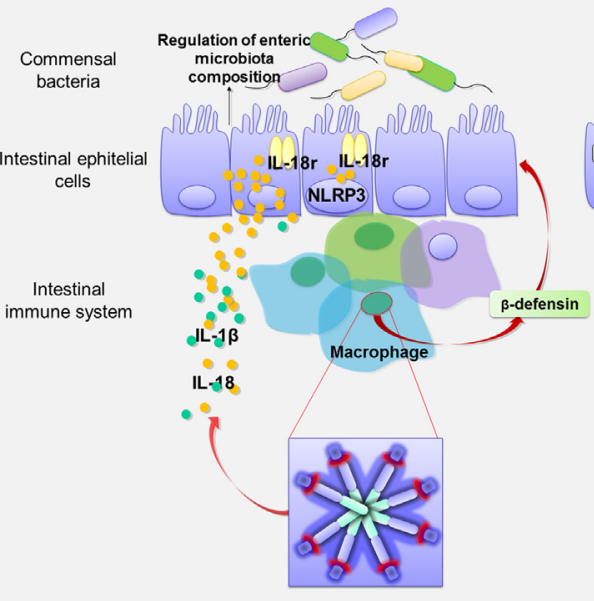

Protective role

Maintenance of the integrity of

intestinal epithelial barrier and

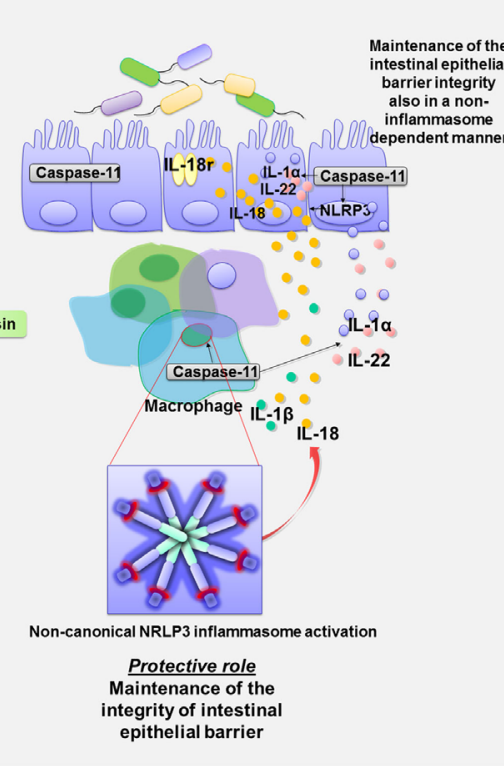

Intestinal inflammation

Canonical NLRP3 inflammasome

Acute

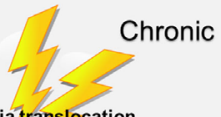

Bacteria

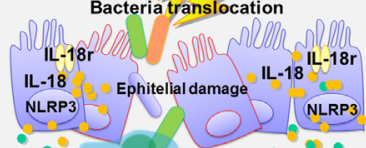

$\therefore \therefore$ (NLRP

$00 \% 80^{\circ}$ Sustaining of the

$\because \because$ Macrophaget $\because \because$

Dendritic cell ${ }^{\circ}$

$\therefore \because \quad \because \%$ Th1 Th17

$\because$ LL-18

$\because \because$

$\because$ IL-13

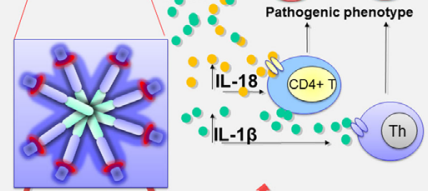

$\therefore$,

\section{RLP3 inflammasome active
Protective role
Tisue repair and \\ Detrimental role \\ Disruption of the intestinal epithel \\ barrier integrity \\ mucosal immune respons}

Non-canonical NLRP3 inflammasome

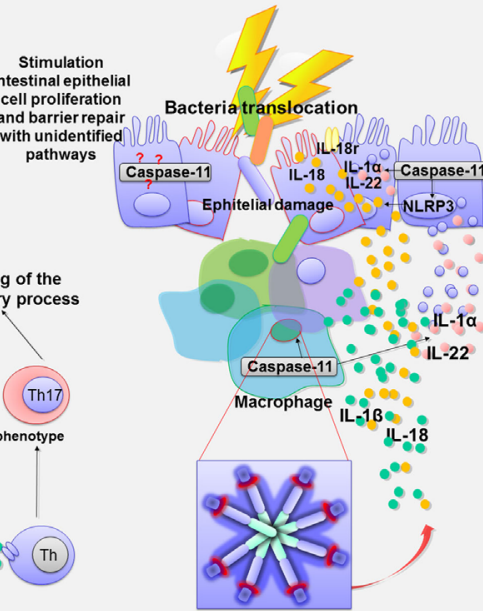

Non-canonical
NRLP3 inflammasome activation Protective role Stimulation intestinal
epithelial cell proliferation and

FIGURE 2 | Canonical and non-canonical activation of nucleotide-binding oligomerization domain leucine rich repeat and pyrin domain-containing protein 3 (NLRP3) inflammasome pathways in intestinal homeostasis and inflammation. Diagram showing the role of canonical and non-canonical activation of NLRP3 inflammasome pathways in intestinal homeostasis and inflammation. Left panel:

Canonical and non-canonical NLRP3 inflammasome activation in intestinal homeostasis. Canonical NLRP3 inflammasome activation plays a key role in the maintenance of the integrity of intestinal epithelial barrier as well as in the enteric microbiota composition through the release of IL-18 by macrophages and intestinal epithelial cells, the regulation of crypt bactericidal capacity, and the release of colonic $\beta$-defensin by macrophages. Likewise, non-canonical NLRP3 inflammasome activation contributes to maintain the integrity of intestinal epithelial barrier through IL-18 release by macrophages and intestinal epithelial cells. In addition, caspase-11 contributes, in a NLRP3-independent manner, to the maintenance of intestinal homeostasis promoting the release of II-1 $\alpha$ and IL-22. Right panel: Canonical and non-canonical NLRP3 inflammasome activation in intestinal inflammation. In the acute phase of inflammation, canonical NLRP3 inflammasome activation promotes the release of IL-1 $\beta$ and IL-18, contributing to tissue repair and maintenance of epithelial barrier integrity. Conversely, in the chronic phase of inflammation, canonical NLRP3 inflammasome overactivation is associated with an increase in IL-1 $\beta$ and IL-18 release that is harmful to the host. In addition, IL-1 $\beta$ and IL-18 release induce the differentiation of T cells into pathogenic Th1 and Th17 phenotypes, which contribute to sustain the inflammatory response. Non-canonical NLRP3 inflammasome activation plays a protective role during bowel inflammation likely via IL-18 release that stimulates intestinal epithelial cell proliferation and barrier repair. In addition, the release of IL-22 and IL-1 $\alpha$ contributes to the repair of intestinal epithelial barrier. However, caspase-11 contributes also to promote intestinal epithelial cell proliferation and barrier repair by recruitment of yet unidentified inflammasomeindependent pathways. 
key role of NLRP3 inflammasome in the maintenance of the integrity of intestinal epithelial barrier as well as in orchestrating the mucosal innate immune response during inflammation. However, further investigations should be implemented to identify the exact mechanism through which NLRP3 modulates the intestinal epithelium-innate immune system interplay both under physiological conditions and in the presence of bowel inflammation.

Besides the regulation of non-immune and immune cells activity, NLRP3 inflammasome influences also the composition of enteric microbiota $(7,38)$. In particular, fecal microbiota in $\mathrm{NLRP}^{-/-}$mice did dramatically differ, in terms of load and species, from WT mice, and such microbial shifts occurred in $N \operatorname{lrp} 3^{-/-}$mice prior to the induction of colitis, suggesting that a reduced inflammasome functionality is associated with enteric bacterial dysbiosis (37). Taken together, these findings expand further available knowledge about the regulatory role of inflammasome in homeostasis, since it appears to coordinate a dynamic interplay between gut microbiota and epithelium-innate immune system, contributing to the maintenance of intestinal microenvironment integrity (Figure 2).

Several lines of evidence point out the concept that deficiencies in NLRP3 inflammasome components can protect mice from DSS-induced colitis $(9,39)$. In particular, in a study by Bauer et al., DSS Nlrp3 $3^{-/-}$mice developed a less severe colitis and produced lower levels of pro-inflammatory cytokines as compared with DSS WT mice. In addition, the pharmacological inhibition of caspase-1 with pralnacasan protected from colonic mucosal damage as with NLRP3 deficiency, suggesting that NLRP3 inflammasome contributes to the pathophysiology of intestinal inflammation and that NLRP3 blockade could represent a viable pharmacological strategy for the management of bowel inflammation $(39,40)$. Different findings about the different roles of NLRP3 inflammasome in bowel inflammation might be ascribed to different experimental conditions. For instance, in reports showing a protective and regulatory action of NLRP3, experiments were performed at the seventh day after 5 days of $3 \%$ DSS treatment and 2 days without DSS exposure, or 7 days of continued 2.5\% DSS exposure. By contrast, in the paper by Bauer et al., describing a detrimental role of NLRP3 in colitis, mice received $2 \%$ DSS for 9 days. It is therefore conceivable that, extending DSS exposure, the overactivation of NLRP3 becomes detrimental for the intestinal microenvironment. Indeed, 6 days after colitis induction, the histopathological score was significantly reduced in DSS NLRP3 ${ }^{-/-}$mice, as compared with DSS WT (39).

Interestingly, in a subsequent paper, Bauer et al. hypothesized a putative role of NLRP3 inflammasome both in the innate and adaptive immune response. In particular, unlike intestinal epithelial cells, where inflammasome-induced IL-18 release promoted a mucosal repair after DSS-induced damage, NLRP3induced IL-18 release from lamina propria macrophages and dendritic cells elicited the activation and differentiation of $\mathrm{CD}^{+}$ $\mathrm{T}$ cells into the Th1 pro-inflammatory phenotype (Figure 2) (41). The relevance of NLRP3 in the modulation of immune cell differentiation was further confirmed by observing that lamina propria dendritic cells in $\mathrm{NLRP}^{-/-}$mice expressed a tolerogenic phenotype $\left(\mathrm{CD}_{103^{+}} \mathrm{DC}\right)$ both under physiological and inflammatory conditions, which may, at least in part, explain the reduced susceptibility of $N \operatorname{lrp} 3^{-/-}$mice to colitis (41). However, the molecular mechanisms through which NLRP3 triggers the differentiation of dendritic cells into a pro-inflammatory phenotype are still unclear and deserve further investigations.

In support of the above results, showing a detrimental role of NLRP3 in bowel inflammation, Zhang et al. (9) observed a causative link between NLRP3 inflammasome activation and development of chronic intestinal inflammation, showing that the increase in colonic IL- $1 \beta$ levels in $I L-10^{-1-}$ mice promoted IL-17 release, known to contribute to the pathogenesis of chronic colitis both in animal models and IBD patients (42). Treatment with IL-1 receptor antagonist or caspase- 1 inhibitors suppressed IL-1 $\beta$ and IL-17 production, thus ameliorating spontaneous colitis in $I L-10^{-/-}$mice. In this setting, it appears that the lack of anti-inflammatory IL-10 cytokine triggers unknown molecular mechanisms that could influence IL-1 $\beta$ release through gene transcription and/or direct regulation of canonical caspase-1-dependent inflammasome activation (9). Based on these findings, the authors hypothesized that, in the absence of anti-inflammatory IL-10 cytokine, intestinal inflammasomes undergo a condition of prolonged activation, leading to an uncontrolled and aberrant inflammasome-mediated immune response that contributes to the development of chronic colitis (9). Therefore, since IL-10 appears to modulate inflammasome activation, an in vivo pharmacological modulation of IL-10 in animal models of hapten-induced colitis could help to unravel the mechanisms underlying the negative regulation of inflammasome by IL-10.

Another considerable issue pertains to the relationship between NLRP3 and gut microbiota. Indeed, Bauer et al. (41) observed that cohousing of $N l r p 3^{-1-}$ mice with WT animals abrogated the protective effect of NLRP3 deficiency during colitis, and increased DSS susceptibility. Based on these results, it is conceivable that changes in enteric bacterial composition and a condition of NLRP3 hypo-functionality could contribute to the pathophysiology of bowel inflammation (41). Therefore, although these findings are in contrast with previous observations, showing a protective role of NLRP3 during colitis (37), both highlight the relevance of NLRP3-enteric microbiota interplay in the maintenance of intestinal homeostasis.

\section{Non-Canonical NLRP3 Inflammasome Activation}

Besides canonical NLRP3 inflammasome activation, over the last years, a pivotal role in the pathophysiology of intestinal inflammation has been proposed also for non-canonical caspase11-dependent NLRP3 inflammasome activation. In particular, caspase-11, widely expressed in both hematopoietic- and nonhematopoietic cells, including macrophages and epithelial cells, once activated by Gram-negative bacteria, promotes NLRP3 inflammasome assembly and subsequent release of IL-1 $\beta$, IL-18 and regulates IL- $1 \alpha$ and HMGB1 release and pyroptosis $(43,44)$.

The contribution of caspase-11 to NLRP3 inflammasome activation has been initially investigated in animal models of acute exposure to enteric bacteria, sepsis, and endotoxic shock 
$(45,46)$. Recently, several lines of evidence have shown that caspase-11-induced inflammasome activation plays a protective role during intestinal inflammation $(8,47)$. In particular,

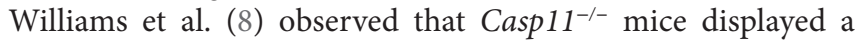
significantly increased morbidity, colonic tissue damage, and leukocyte infiltration following DSS exposure, thus suggesting an increased susceptibility to DSS-induced colitis that was ascribed to a decrease in colonic inflammasome-induced

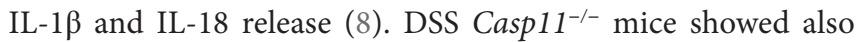
a significant increase in caspase- 1 expression, which, however, was not associated with an increased inflammasome activity (8). In support of the protective role of caspase-11, it has been observed that both chimeric Casp11 $1^{-/-}$mice, receiving WT bone marrow (WT $\rightarrow$ Casp11 $1^{-/}$), and chimeric WT mice, receiving

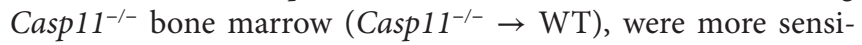
tive to DSS-induced colitis as compared to WT mice receiving WT bone marrow. In particular, chimeric WT $\rightarrow$ Casp $11^{-/-}$or Casp $11^{-/-} \rightarrow$ WT DSS mice displayed a significantly increased histopathological damage, epithelial tethering, large areas of erosion, extensive areas of ulceration, enhanced inflammatory cell infiltration, and increased crypt atrophy as compared with chimeric DSS WT $\rightarrow$ WT animals (8). A proposed mechanism, underlying the protective role of caspase-11-induced inflammasome activation, calls into play its ability of regulating the release of IL-18, IL-22, and IL-1 $\alpha$ cytokines, known to promote intestinal epithelial cell proliferation and barrier repair (47). Indeed, Casp $11^{-/-}$DSS mice showed reduced colonic IL-18, IL-22, and IL-1 $\alpha$ levels in comparison with WT DSS animals, thus suggesting that decreased levels of these cytokines, in particular IL-18, could contribute to the increase in epithelial barrier permeability, with consequent bacterial translocation into the lamina propria and exacerbation of the inflammatory response (48-50). Taken together, these findings suggest that caspase-11, via non-canonical inflammasome activation, regulates mucosal and epithelial barrier integrity during intestinal inflammation by increasing epithelial cell proliferation and inhibiting cell death (Figure 2).

The protective role of caspase-11-induced non-canonical NLRP3 inflammasome activation has been shown in a model of Gram-negative C. rodentium infection-induced colitis (51). In particular, in autophagy defective mice, made knock out for nucleotide-binding oligomerization domain-like receptors NLRs (NOD2) and recruit receptor interacting protein 2, infected with $C$. rodentium, the increase in oxidative stress activated the c-Jun N-terminal kinase (JNK) signaling that, in turn, increased caspase-11 expression and non-canonical NLRP3 inflammasome activation, with consequent protection of colonic epithelial barrier (51). These results corroborate previous findings, supporting the regulatory role of non-canonical NLRP3 inflammasome activation in the maintenance of intestinal homeostasis, and, most importantly, they show that, besides the TLR4-TRIF-IFN- $\beta$ pathway, JNK signaling promotes non-canonical inflammasome activation. In this context, despite the observation that JNK signaling can be activated also by TLR stimulation (52), it is not clear whether the stimulation of TLR-MyD88-JNK pathway promotes caspase-11-dependent non-canonical NLRP3 inflammasome activation during intestinal inflammation. Therefore, there is still need to evaluate the role of this pathway in animal models of colitis.

At odds with the above data, Demon et al. (53) suggested that sensitivity to DSS colitis in Casp $11^{-/-}$mice is independent from caspase-1-induced canonical inflammasome activation, since the colonic levels of IL-1 $\beta$ and IL-18, as well as circulating HMGB1 in DSS Casp11 $11^{-1-}$ mice, did not differ from DSS WT mice, suggesting that caspase- 11 protects against colitis independently from inflammasome activation and, therefore, hypothesizing an unidentified pathway for caspase-11 in bowel inflammation (Figure 2) (53). These conflicting findings about the relevance of caspase-11-induced non-canonical inflammasome activation could result from different experimental designs, as well as from environmental variability. For instance, since caspase-11 is activated by Gram-negative bacteria, changes in gut microbiota composition could (i) influence hypo- or hyper-activation of caspase-11-induced non-canonical NLRP3 inflammasome, (ii) alter basal IFN production $(54,55)$, with subsequent changes in caspase-11 expression and/or function, or (iii) trigger yet unidentified molecular NLRP3-independent pathways involved in the pathophysiology of gut inflammation.

\section{Discussion}

Current data allow to hypothesize that NLRP3 inflammasome can play both protective and detrimental roles in bowel inflammation, depending on the choice of colitis models and variations of commensal enteric microflora. In particular, in the model of DSS-induced acute colitis, which causes a direct damage to the epithelial barrier, with consequent stimulation of innate immune cells by commensal bacteria, infiltration of myeloid cells and massive inflammation, IL-1 $\beta$ and IL-18 appear to be essential for tissue repair and the maintenance of epithelial barrier integrity (56), thus suggesting a protective role of NLRP3. By contrast, in animal models of chronic colitis, the inflammasome-induced IL- $1 \beta$ release induces differentiation of $\mathrm{T}$ cells into pathogenic Th17 phenotypes, thus contributing to sustain the inflammatory process (9). Despite these conflicting and heterogeneous findings, it appears that, in the first acute phase of inflammation, the NLRP3 inflammasome acts as a key player to restore intestinal homeostasis. Conversely, in chronic colitis, where a disruption of the intestinal epithelial barrier and an exacerbation of the mucosal immune response occur, the overactivation of NLRP3 inflammasome results to be harmful to the host (Figure 2). However, the results from knockout mouse models, where there is a complete removal of NLRP3 protein complex, cannot be easily and fully translated into the clinical setting, since NLRP3 gene deletion might trigger unknown compensatory immune mechanisms that influence the disease outcome. Furthermore, it is also noteworthy that in animals with complete deletion of NLRP3 gene no distinction between canonical and/or non-canonical NLRP3 inflammasome activation can be made. Therefore, the in vivo pharmacological modulation of canonical NLRP3 inflammasome in more predictive animal models of colitis should be investigated, in order to clarify the role of this enzymatic complex in the pathophysiology of bowel inflammation. Data on the effects stemming from the pharmacological modulation of NLRP3 inflammasome are discussed in the following sections. 
With regard to non-canonical NLRP3 inflammasome activation, the majority of current data suggest that caspase11-dependent NLRP3 activation, although dispensable for caspase-1-inflammasome assembly, contributes to protection against DSS-induced colitis regulating the epithelial barrier integrity. However, owing to scarce, conflicting and heterogeneous findings, it remains unclear whether caspase-11, expressed in the colonic mucosa, plays a protective role also in intestinal inflammation independently from canonical NLRP3 inflammasome activation. Accordingly, there is a strong need for further experiments, aimed at evaluating how the simultaneous caspase-1 and caspase-11 gene deletion, as well as the pharmacological modulation of caspase-11, could interfere with NLRP3 assembly and consequently with the pathophysiology of bowel inflammation. In addition, given the relevance of intestinal microbiota in caspase-11 activation, extensive investigations are needed to evaluate whether changes in enteric bacteria composition could influence caspase-11 activity and consequent non-canonical inflammasome assembly.

\section{PHARMACOLOGICAL MODULATION OF NLRP3 INFLAMMASOME IN BOWEL INFLAMMATION}

The involvement of inflammasome pathways in the pathophysiology of intestinal inflammation is fostering research on the potential therapeutic benefits, in terms of anti-inflammatory activity, resulting from the pharmacological targeting of NLRP3 inflammasome. At present, the majority of available studies have investigated the role of NLRP3 in several experimental models of colitis, displaying remarkable beneficial effects by the pharmacological modulation of this enzymatic complex (57-59). In particular, DSS-induced colitis has been largely employed, since in this model lysosomal damage and increased ROS levels can lead to an overactivation of NLRP3 inflammasome (39). Following DSS administration, surface molecules produced by microorganisms or other inflammatory factors (i.e., LPS) can promote also the first step of NLRP3 assembly, through the activation of NF- $\kappa B$ transcription, with subsequent increase in NLRP3 as well as pro-IL-1 $\beta$ and pro-IL-18 protein levels. Furthermore, DSS treatment is associated with an increase in extracellular ATP or bacterial toxins, which are able to stimulate caspase- 1 activation directly, thereby releasing IL-1 $\beta$ and IL-18 $(39,58)$. Accordingly, several targets have been identified for inhibiting the assembly of NLRP3 (Figure 1).

A pioneering study by Dashdorj et al. (59) showed that MitoQ, a mitochondria-targeted derivative of the antioxidant ubiquinone, endowed with antioxidant and anti-apoptotic properties, exerted beneficial effects on experimental colitis through a decrease in colonic NLRP3 and caspase-1 expression, with consequent decrease in IL- $1 \beta$ and IL-18 release (59). The molecular mechanism underlying NLRP3 inflammasome blockade was proposed to depend on the ability of MitoQ to suppress ROS-induced dissociation of thioredoxin-interacting protein (TXNIP), from thioredoxin, thus inhibiting the interaction of TXNIP with NLRP3 (59). Indeed, although Masters et al. (60) showed that TXNIP is not essential to NLRP3 activation in bone marrow-derived macrophages primed with LPS and then stimulated with $S$. aureus, silica, or ATP, in the setting of colitis, where an increase in oxidative stress and activation of different inflammatory pathways occur, the dissociation of TXNIP could represent one of the mechanisms underlying NLRP3 activation (59).

The inhibition of mitochondrial ROS generation, as a suitable pharmacological target for inhibiting NLRP3 inflammasome assembly, has been confirmed by a subsequent study, showing that in vivo administration of levornidazole, the levo isomer of ornidazole generally used for protozoan infections, to DSS mice exerted enteric anti-inflammatory effects through the blockade of NLRP3 inflammasome assembly by suppression of ROS generation. These findings suggest that the blockade of NLRP3 upstream signaling could represent a suitable pharmacological target for the management of intestinal inflammation (61).

Consistent with the above data, two recent papers by Wang et al. (58) and Liu et al. (62) have reported that the inhibition of ROS formation exerted beneficial effects in colitis through the blockade of NLRP3 assembly $(58,62)$. In particular, these authors observed that two small molecules, 3-(2-oxo2-phenylethylidene)-2,3,6,7-tetrahydro-1H-pyrazino-[2,1-a] isoquinolin-4(11bH)-one (compound 1$)$ and dimethyl fumarate, promote the transcription of genes coding for various detoxification and antioxidant enzymes, through the activation of NFE-related factor 2 (Nrf2). The subsequent inhibition of ROS formation has been shown to exert inhibitory effects on NLRP3 assembly $(58,62)$. Indeed, after exposure to environmental or intracellular stresses, such as ROS, Nrf2 translocates into the nucleus and binds to antioxidant response elements (AREs), which in turn induce the production of cytoprotective enzymes, such as heme oxygenase $1, \mathrm{NAD}(\mathrm{P}) \mathrm{H}$ quinine oxidoreductases, and glutathione $S$-transferases, that are pivotal to maintain optimal cellular functions (63). In this respect, the stimulation of Nrf2/ARE pathway could represent an indirect molecular target to inhibit NLRP3 inflammasome activation. However, further extensive investigations are needed to characterize the actual molecular mechanisms underlying the Nrf2-ROS-NLRP3 interplay and, most importantly, the correlation between Nrf2 stimulation and NLRP3 inhibition in the setting of bowel inflammation.

Besides targeting the inflammasome upstream signaling, NLRP3 blockade via caspase-1 inhibition has been shown also to exert anti-inflammatory effects in DSS mice $(57,64)$. In particular, a synthetic benzimidazole derivative and fumigaclavine C, a fungal metabolite, through the inhibition of caspase-1 activation, exerted beneficial effects on colonic inflammation reducing protein and mRNA levels of colonic TNF, IL-1 $\beta$, and IL-17 pro-inflammatory cytokines $(57,64)$. However, even if both compounds have caspase-1 inhibition as ultimate goal, they influence intracellular signaling in different ways. Indeed, the benzimidazole derivative has been shown to inhibit MAPK and STAT1 signaling without interaction with NF- $\mathrm{KB}$-mediated transcription, while fumigaclavine $\mathrm{C}$ was found to significantly

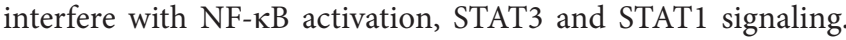


These findings suggest that the inhibition of the primary TRLMyD88-NF- $\mathrm{B}$ step involved in NLRP3 inflammasome activation, though at different steps of the intracellular cascade, represents a suitable pharmacological target for inhibiting the NLRP3 assembly, and therefore a promising strategy for treatment of bowel inflammation.

In further support of the above data, a recent paper by Liu et al. (65) showed that the inhibition of mucosa-associatedlymphoid-tissue lymphoma-translocation gene 1 (MALT1), a scaffold protein, which recruits the IкB kinase complex leading to release and activation of $\mathrm{NF}-\kappa \mathrm{B}$, ameliorated clinical symptoms and histopathologic features of DSS-induced colitis through NF- $\kappa$ B and NLRP3 inhibition, thus interfering with both inflammasome activation steps $(65,66)$. In particular, treatment with two specific MALT1 inhibitors, MI-2 and mepazine, dosedependently attenuated the symptoms of colitis in mice through a decrease in protein and mRNA levels of colonic TNF, IL-1 $\beta$, IL-6, IL-18, IL-17A, and IFN- $\gamma$ pro-inflammatory cytokines (66). The mechanisms underlying the inhibitory effects of MALT1 in DSSinduced colitis have been ascribed to the inhibition on NF- $\kappa$ B and NLRP3 inflammasome activation in macrophages, thus implying that MALT1-NF- $\mathrm{KB}$ signaling regulates NLRP3 inflammasome activation. However, since the NF- $\kappa B$ pathway is involved in the transcription of both pro- and anti-inflammatory mediators (i.e., IL-10 and TGF- $\beta$ ), extensive investigations are required to identify more selective targets to inhibit NLRP3 inflammasome oligomerization, thereby counteracting the pathophysiological events underlying NLRP3 activation, and attenuating bowel inflammation.

Of interest, several lines of evidence have shown that various NLRP3-targeting natural compounds are able to exert antiinflammatory effects on DSS-induced colitis in mice. Guo et al. (66) observed that oral administration of asiatic acid, a natural triterpenoid compound, dose-dependently attenuated body weight loss, shortening of colon length, histopathologic scores, myeloperoxidase activity, and colonic TNF, IL-1 $\beta$, IL-6, and IFN$\gamma$ levels in mice with DSS-induced colitis through the inhibition of NLRP3 inflammasome activation (67). In particular, the authors found that asiatic acid inhibited the upstream signaling of inflammasome oligomerization by suppressing mitochondrial ROS generation, as well as caspase- 1 activation and inflammasome assembly. Likewise, treatment with fraxinellone, a natural lactone endowed with immunosuppressive activity, significantly reduced weight loss, diarrhea and colonic macroscopic damage, as well as myeloperoxidase, alkaline phosphatase, and colonic TNF, IL-1 $\beta$, IL-6, and IL-18 levels in DSS-induced colitis mice (68). Such anti-inflammatory effects were ascribed to the inhibition of $\mathrm{CD} 1 \mathrm{~b}^{+}$macrophage infiltration, as well as the decrease in mRNA levels for colonic macrophage-related proteins, including intercellular adhesion molecule 1 (ICAM1), vascular cell adhesion molecule 1 (VCAM1), iNOS, and cyclooxygenase-2 (COX-2), through NF- $\kappa \mathrm{B}$ signaling and NLRP3 inhibition. These findings represent a point of novelty, since they support the view that the blockade of NLRP3 assembly can influence also the activation of infiltrating macrophages by inhibiting the release of intercellular adhesion molecules and pro-inflammatory mediators contributing to the inflammatory process.
Recent evidence has shown that several flavonoid derivatives

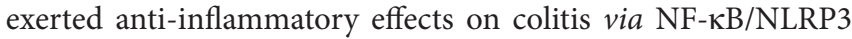
inhibition (69-71). In particular, treatment with wogonoside, a glucuronide metabolite of the bioactive flavonoid wogonin, reduced significantly colonic NF- $\mathrm{KB}$ and NLRP3 expression, as well as caspase- 1 expression and activity in mice with colitis, exerting beneficial effects on colonic inflammation (69). Likewise, the administration of alpinetin, a novel plant flavonoid isolated from Alpinia katsumadai Hayata, significantly attenuated diarrhea, colonic shortening, histological damage, and myeloperoxidase activity as well as colonic TNF and IL- $1 \beta$ expression in mice with DSS-induced colitis, likely by suppressing TRL4-NF- $\kappa \mathrm{B}$ and NLRP3-ASC-caspase-1 signaling (70). However, the authors documented the ability of alpinetin of inhibiting NLRP3 activation in in vitro THP-1 cells, omitting the evaluation of alpinetin effects on NLRP3 activation in DSS mice.

The protective effects of flavonoids via NLRP3 inhibition have been shown also in a mouse model of DSS-induced colitis (71). In particular, a dietary apigenin (API) enrichment decreased the macroscopic and microscopic signs of colitis and reduced colonic PGE, COX-2, and iNOS expressions as well as serum matrix metalloproteinase (MMP-3) levels. In addition, API diet reduced IL-1 $\beta$ and TNF pro-inflammatory cytokine release in primary LPS-stimulated splenocytes. The beneficial effects of API on colonic inflammation result from the inhibition of both canonical and non-canonical NLRP3 inflammasome pathways, through the regulation of caspase- 1 and caspase-11 enzyme expression and activity (71). Indeed, although caspase-11 has been proposed to mediate a protective role in the host during the acute phases of colitis, it appears to be detrimental in chronic inflammation, where it is significantly upregulated and promotes IL- $1 \beta$ and IL-18 release $(8,47)$. These findings demonstrate, for the first time, that the pharmacological blockade of both canonical and non-canonical NLRP3 activation could represent a suitable and promising pharmacological target for treatment of bowel inflammation. Nevertheless, the molecular mechanisms through which API can block canonical and non-canonical NLRP3 assembly remain to be clarified.

\section{OVERALL CONCLUSION AND FUTURE DIRECTIONS}

Studies aimed at characterizing the molecular mechanisms and downstream signaling underlying the canonical and noncanonical NLRP3 inflammasome activation have unraveled the pivotal and dual role of this enzymatic complex in the intestinal homeostasis. According to current information, NLRP3 regulates the integrity of intestinal mucosal barrier under physiological conditions, but it can shape also the immune response against commensal microbiota during bowel inflammation.

One considerable deficiency in our knowledge concerns how canonical and non-canonical NLRP3 inflammasome coordinate differently the dynamic interplays among gut microbiotaepithelium-innate immune system. For instance, it remains unclear whether alterations of enteric bacteria composition promote an abnormal caspase-1- and/or caspase-11-dependent NLRP3 inflammasome activation, or whether, vice versa, the 
overactivation of NLRP3 alters epithelial barrier integrity and gut microbiota with consequent alteration of intestinal homeostasis. In this context, given the high complexity of the NLRP3 inflammasome system, strong efforts are needed to better understand how canonical and non-canonical NLRP3 manage their downstream signaling to maintain or break down intestinal homeostasis. Nevertheless, despite current heterogeneous and conflicting evidence, beneficial effects resulting from the pharmacological modulation of NLRP3 in animal models of colitis have led to postulate that the overactivation of NLRP3 during bowel inflammation is detrimental for the host. Therefore, the blockade of NLPR3 activation could represent a suitable pharmacological approach for the management of inflammatory intestinal disorders.

A recent study by Coll et al. (72) showed that the pharmacological blockade of canonical and non-canonical NLRP3 activation with MCC950, a recognized selective, small molecule inhibitor of NLRP3, reduced IL-1 $\beta$ tissue levels and attenuated the severity of experimental autoimmune encephalomyelitis, an animal model of multiple sclerosis (72). Furthermore, MCC950 rescued neonatal lethality in a mouse model of CAPS, and it was effective in reducing IL-1 $\beta$ levels in human peripheral blood mononuclear cells from patients with Muckle-Wells syndrome, thus suggesting its putative therapeutic activity in NLRP3-associated syndromes, including autoinflammatory and autoimmune diseases (72). Based on these observations, testing MCC950 in animals with colitis (i.e., DSS, TNBS, or $I L-10^{-/-}$) would allow a better characterization of the anti-inflammatory effects resulting from selective inhibition of canonical and non-canonical NLRP3 inflammasome activation.

Another relevant issue concerns the inhibition of NLRP3 downstream signaling through the blockade of IL- $\beta$ receptor. In this respect, several lines of evidence have shown that treatment with IL- $\beta$ receptor antagonists (i.e., anakinra) exerted beneficial effects in patients with immune-mediated inflammatory diseases (i.e., rheumatoid arthritis, ankylosing spondylitis, and gout) (73). In addition, anakinra reduced postoperative inflammation and ameliorated postoperative ileus in mice (74), thus suggesting that IL- $1 \beta$ receptor blockade exerts beneficial effects during intestinal inflammation. Based on these findings, it is conceivable that both upstream and downstream inhibition of NLRP3 inflammasome could represent suitable pharmacological approaches for treatment of bowel inflammation. Therefore, the

\section{REFERENCES}

1. De Nardo D, Latz E. NLRP3 inflammasomes link inflammation and metabolic disease. Trends Immunol (2011) 32(8):373-9. doi:10.1016/j.it.2011. 05.004

2. Broderick L, De Nardo D, Franklin BS, Hoffman HM, Latz E. The inflammasomes and autoinflammatory syndromes. Annu Rev Pathol (2015) 10:395-424. doi:10.1146/annurev-pathol-012414-040431

3. Elinav E, Henao-Mejia J, Flavell RA. Integrative inflammasome activity in the regulation of intestinal mucosal immune responses. Mucosal Immunol (2013) 6(1):4-13. doi:10.1038/mi.2012.115

4. Gagliani N, Palm NW, de Zoete MR, Flavell RA. Inflammasomes and intestinal homeostasis: regulating and connecting infection, inflammation possible effects of the pharmacological blockade of IL- $1 \beta$ receptor in different animal models of colitis should be investigated, in order to find out the better strategy to inhibit the NLRP3 inflammasome pathway and counteract bowel inflammation.

In support of preclinical findings, clinical evidence has documented an increased IL- $1 \beta$ secretion from colonic tissues and macrophages of IBD patients, these patterns being correlated with the severity of disease, thus suggesting IL-1 $\beta$ as a key pro-inflammatory cytokine for the pathogenesis of IBDs (75). In addition, the activation of NLRP3 inflammasome in monocytes infiltrating the lamina propria and M1 pro-inflammatory macrophages isolated from intestinal specimens of IBD patients, seems to contribute to the disruption of epithelial barrier through a deregulation of tight junction proteins (i.e., claudin-1, claudin-2, and junctional adhesion molecule-A), as well as to induce epithelial cell apoptosis (76). In particular, NLRP3 inflammasome-induced IL-1 $\beta$ and IL-18 release from monocytes infiltrating the lamina propria alters tight junctions and promotes apoptosis in intestinal epithelial cells, and, subsequently M1 macrophages, recruited into the lamina propria, contribute to sustain the immune innate response, thus suggesting a detrimental role of NLRP3 inflammasome in IBD patients (76). Accordingly, a translation of preclinical evidence into clinical practice could allow a better understanding of protective/detrimental shift of NLRP3 in IBD patients.

In conclusion, given the heterogeneity of preclinical studies and the paucity of human studies, extensive investigations are awaited for better understanding the NLRP3 inflammasome functionality in non-immune and immune cells since early stages of intestinal inflammation, in order to clarify the relevance of NLRP3 inflammasome in the pathophysiology of IBDs. In addition, considering the close inflammasome-gut microbiota interplay, and that genetic NLRP3 mutations and environmental factors, altering the gut microbiota, are involved in the pathogenesis of IBDs, future investigations should be addressed to characterize the correlation between changes in enteric bacteria composition and hypo- or hyperfunctionality of NLRP3 inflammasome.

\section{AUTHOR CONTRIBUTIONS}

CP, GL-C, and LA wrote the first draft of the manuscript. CP prepared the figures. $\mathrm{CB}$ and $\mathrm{MF}$ revised the manuscript.

and the microbiota. Int Immunol (2014) 26(9):495-9. doi:10.1093/intimm/ dxu066

5. Kayagaki N, Warming S, Lamkanfi M, Vande Walle L, Louie S, Dong J, et al. Non-canonical inflammasome activation targets caspase-11. Nature (2011) 479(7371):117-21. doi:10.1038/nature10558

6. Lissner D, Siegmund B. The multifaceted role of the inflammasome in inflammatory bowel diseases. ScientificWorldJournal (2011) 11:1536-47. doi:10.1100/tsw.2011.139

7. Hirota SA, Ng J, Lueng A, Khajah M, Parhar K, Li Y, et al. NLRP3 inflammasome plays a key role in the regulation of intestinal homeostasis. Inflamm Bowel Dis (2011) 17(6):1359-72. doi:10.1002/ibd.21478

8. Williams TM, Leeth RA, Rothschild DE, McDaniel DK, Coutermarsh-Ott SL, Simmons AE, et al. Caspase-11 attenuates gastrointestinal inflammation 
and experimental colitis pathogenesis. Am J Physiol Gastrointest Liver Physiol (2015) 308(2):G139-50. doi:10.1152/ajpgi.00234.2014

9. Zhang J, Fu S, Sun S, Li Z, Guo B. Inflammasome activation has an important role in the development of spontaneous colitis. Mucosal Immunol (2014) 7(5):1139-50. doi:10.1038/mi.2014.1

10. Latz E, Xiao TS, Stutz A. Activation and regulation of the inflammasomes. Nat Rev Immunol (2013) 13(6):397-411. doi:10.1038/nri3452

11. Bauernfeind FG, Horvath G, Stutz A, Alnemri ES, MacDonald K, Speert $\mathrm{D}$, et al. Cutting edge: NF-kappaB activating pattern recognition and cytokine receptors license NLRP3 inflammasome activation by regulating NLRP3 expression. JImmunol (2009) 183(2):787-91. doi:10.4049/ jimmunol.0901363

12. Faustin B, Lartigue L, Bruey JM, Luciano F, Sergienko E, Bailly-Maitre B, et al. Reconstituted NALP1 inflammasome reveals two-step mechanism of caspase-1 activation. Mol Cell (2007) 25(5):713-24. doi:10.1016/j.molcel. 2007.01.032

13. Mariathasan S, Weiss DS, Newton K, McBride J, O'Rourke K, Roose-Girma $\mathrm{M}$, et al. Cryopyrin activates the inflammasome in response to toxins and ATP. Nature (2006) 440(7081):228-32. doi:10.1038/nature04515

14. Compan V, Baroja-Mazo A, López-Castejón G, Gomez AI, Martínez $\mathrm{CM}$, Angosto D, et al. Cell volume regulation modulates NLRP3 inflammasome activation. Immunity (2012) 37(3):487-500. doi:10.1016/ j.immuni.2012.06.013

15. Halle A, Hornung V, Petzold GC, Stewart CR, Monks BG, Reinheckel T, et al. The NALP3 inflammasome is involved in the innate immune response to amyloid-beta. Nat Immunol (2008) 9(8):857-65. doi:10.1038/ni.1636

16. Lopez-Castejon G, Luheshi NM, Compan V, High S, Whitehead RC, Flitsch $\mathrm{S}$, et al. Deubiquitinases regulate the activity of caspase-1 and interleukin-1beta secretion via assembly of the inflammasome. J Biol Chem (2013) 288(4):2721-33. doi:10.1074/jbc.M112.422238

17. Munoz-Planillo R, Kuffa P, Martínez-Colón G, Smith BL, Rajendiran TM, Núñez G. $\mathrm{K}(+)$ efflux is the common trigger of NLRP3 inflammasome activation by bacterial toxins and particulate matter. Immunity (2013) 38(6):1142-53. doi:10.1016/j.immuni.2013.05.016

18. Murakami T, Ockinger J, Yu J, Byles V, McColl A, Hofer AM, et al. Critical role for calcium mobilization in activation of the NLRP3 inflammasome. Proc Natl Acad Sci U S A (2012) 109(28):11282-7. doi:10.1073/ pnas.1117765109

19. Aachoui Y, Sagulenko V, Miao EA, Stacey KJ. Inflammasome-mediated pyroptotic and apoptotic cell death, and defense against infection. Curr Opin Microbiol (2013) 16(3):319-26. doi:10.1016/j.mib.2013. 04.004

20. Martin-Rodriguez S, Caballo C, Gutierrez G, Vera M, Cruzado JM, Cases A, et al. TLR4 and NALP3 inflammasome in the development of endothelial dysfunction in uraemia. Eur J Clin Invest (2015) 45(2):160-9. doi:10.1111/eci.12392

21. Casson CN, Shin S. Inflammasome-mediated cell death in response to bacterial pathogens that access the host cell cytosol: lessons from Legionella pneumophila. Front Cell Infect Microbiol (2013) 3:111. doi:10.3389/fcimb.2013. 00111

22. Kayagaki N, Stowe IB, Lee BL, O'Rourke K, Anderson K, Warming S, et al. Caspase-11 cleaves gasdermin D for non-canonical inflammasome signalling. Nature (2015) 526(7575):666-71. doi:10.1038/nature15541

23. Shi J, Zhao Y, Wang K, Shi X, Wang Y, Huang H, et al. Cleavage of GSDMD by inflammatory caspases determines pyroptotic cell death. Nature (2015) 526(7575):660-5. doi:10.1038/nature15514

24. Lopez-Castejon G, Pelegrin P. Current status of inflammasome blockers as anti-inflammatory drugs. Expert Opin Investig Drugs (2012) 21(7):995-1007. doi:10.1517/13543784.2012.690032

25. Lu B, Wang H, Andersson U, Tracey KJ. Regulation of HMGB1 release by inflammasomes. Protein Cell (2013) 4(3):163-7. doi:10.1007/s13238-0122118-2

26. Lamkanfi M, Sarkar A, Vande Walle L, Vitari AC, Amer AO, Wewers MD, et al. Inflammasome-dependent release of the alarmin HMGB1 in endotoxemia. J Immunol (2010) 185(7):4385-92. doi:10.4049/jimmunol.1000803

27. Gurung P, Malireddi RK, Anand PK, Demon D, Vande Walle L, Liu Z, et al. Toll or interleukin-1 receptor (TIR) domain-containing adaptor inducing interferon-beta (TRIF)-mediated caspase-11 protease production integrates toll-like receptor 4 (TLR4) protein- and Nlrp3 inflammasome-mediated host defense against enteropathogens. J Biol Chem (2012) 287(41):34474-83. doi:10.1074/jbc.M112.401406

28. Sander LE, Davis MJ, Boekschoten MV, Amsen D, Dascher CC, Ryffel B, et al. Detection of prokaryotic mRNA signifies microbial viability and promotes immunity. Nature (2011) 474(7351):385-9. doi:10.1038/nature10072

29. Schauvliege R, Vanrobaeys J, Schotte P, Beyaert R. Caspase-11 gene expression in response to lipopolysaccharide and interferon-gamma requires nuclear factor-kappa B and signal transducer and activator of transcription (STAT) 1. J Biol Chem (2002) 277(44):41624-30. doi:10.1074/jbc. M207852200

30. Broz P, Ruby T, Belhocine K, Bouley DM, Kayagaki N, Dixit VM, et al. Caspase-11 increases susceptibility to Salmonella infection in the absence of caspase-1. Nature (2012) 490(7419):288-91. doi:10.1038/nature11419

31. Rathinam VA, Vanaja SK, Waggoner L, Sokolovska A, Becker C, Stuart LM, et al. TRIF licenses caspase-11-dependent NLRP3 inflammasome activation by gram-negative bacteria. Cell (2012) 150(3):606-19. doi:10.1016/ j.cell.2012.07.007

32. Vigano E, Mortellaro A. Caspase-11: the driving factor for noncanonical inflammasomes. Eur J Immunol (2013) 43(9):2240-5. doi:10.1002/ eji. 201343800

33. Shi J, Zhao Y, Wang Y, Gao W, Ding J, Li P, et al. Inflammatory caspases are innate immune receptors for intracellular LPS. Nature (2014) 514(7521):18792. doi:10.1038/nature 13683

34. Maynard CL, Elson CO, Hatton RD, Weaver CT. Reciprocal interactions of the intestinal microbiota and immune system. Nature (2012) 489(7415):231-41. doi:10.1038/nature11551

35. Camilleri M, Madsen K, Spiller R, Greenwood-Van Meerveld B, Verne GN. Intestinal barrier function in health and gastrointestinal disease. Neurogastroenterol Motil (2012) 24(6):503-12. doi:10.1111/ j.1365-2982.2012.01921.x

36. Rescigno $\mathrm{M}$. The intestinal epithelial barrier in the control of homeostasis and immunity. Trends Immunol (2011) 32(6):256-64. doi:10.1016/j.it.2011. 04.003

37. Zaki MH, Boyd KL, Vogel P, Kastan MB, Lamkanfi M, Kanneganti TD. The NLRP3 inflammasome protects against loss of epithelial integrity and mortality during experimental colitis. Immunity (2010) 32(3):379-91. doi:10.1016/ j.immuni.2010.03.003

38. Dupaul-Chicoine J, Yeretssian G, Doiron K, Bergstrom KS, McIntire CR, LeBlanc PM, et al. Control of intestinal homeostasis, colitis, and colitis-associated colorectal cancer by the inflammatory caspases. Immunity (2010) 32(3):367-78. doi:10.1016/j.immuni.2010.02.012

39. Bauer C, Duewell P, Mayer C, Lehr HA, Fitzgerald KA, Dauer M, et al. Colitis induced in mice with dextran sulfate sodium (DSS) is mediated by the NLRP3 inflammasome. Gut (2010) 59(9):1192-9. doi:10.1136/gut.2009. 197822

40. Loher F, Bauer C, Landauer N, Schmall K, Siegmund B, Lehr HA, et al. The interleukin- 1 beta-converting enzyme inhibitor pralnacasan reduces dextran sulfate sodium-induced murine colitis and T helper $1 \mathrm{~T}$-cell activation. J Pharmacol Exp Ther (2004) 308(2):583-90. doi:10.1124/jpet.103. 057059

41. Bauer C, Duewell P, Lehr HA, Endres S, Schnurr M. Protective and aggravating effects of Nlrp3 inflammasome activation in IBD models: influence of genetic and environmental factors. Dig Dis (2012) 30(Suppl 1):82-90. doi:10.1159/000341681

42. Sutton CE, Lalor SJ, Sweeney CM, Brereton CF, Lavelle EC, Mills KH. Interleukin-1 and IL-23 induce innate IL-17 production from gammadelta $\mathrm{T}$ cells, amplifying Th17 responses and autoimmunity. Immunity (2009) 31(2):331-41. doi:10.1016/j.immuni.2009.08.001

43. Vanaja SK, Rathinam VA, Fitzgerald KA. Mechanisms of inflammasome activation: recent advances and novel insights. Trends Cell Biol (2015) 25(5):308-15. doi:10.1016/j.tcb.2014.12.009

44. Eldridge MJ, Shenoy AR. Antimicrobial inflammasomes: unified signalling against diverse bacterial pathogens. Curr Opin Microbiol (2015) 23:32-41. doi:10.1016/j.mib.2014.10.008

45. Hagar JA, Powell DA, Aachoui Y, Ernst RK, Miao EA. Cytoplasmic LPS activates caspase-11: implications in TLR4-independent endotoxic shock. Science (2013) 341(6151):1250-3. doi:10.1126/science.1240988

46. Knodler LA, Crowley SM, Sham HP, Yang H, Wrande M, Ma C, et al. Noncanonical inflammasome activation of caspase-4/caspase-11 mediates 
epithelial defenses against enteric bacterial pathogens. Cell Host Microbe (2014) 16(2):249-56. doi:10.1016/j.chom.2014.07.002

47. Oficjalska K, Raverdeau M, Aviello G, Wade SC, Hickey A, Sheehan $\mathrm{KM}$, et al. Protective role for caspase-11 during acute experimental murine colitis. J Immunol (2015) 194(3):1252-60. doi:10.4049/jimmunol. 1400501

48. Siegmund B. Interleukin-18 in intestinal inflammation: friend and foe? Immunity (2010) 32(3):300-2. doi:10.1016/j.immuni.2010.03.010

49. Sonnenberg GF, Fouser LA, Artis D. Functional biology of the IL-22IL-22R pathway in regulating immunity and inflammation at barrier surfaces. Adv Immunol (2010) 107:1-29. doi:10.1016/B978-0-12-381300-8. 00001-0

50. Tamboli CP, Neut C, Desreumaux P, Colombel JF. Dysbiosis in inflammatory bowel disease. Gut (2004) 53(1):1-4. doi:10.1136/gut.53.1.1

51. Lupfer CR, Anand PK, Liu Z, Stokes KL, Vogel P, Lamkanfi M, et al. Reactive oxygen species regulate caspase-11 expression and activation of the non-canonical NLRP3 inflammasome during enteric pathogen infection. PLoS Pathog (2014) 10(9):e1004410. doi:10.1371/journal.ppat. 1004410

52. Takeda K, Akira S. TLR signaling pathways. Semin Immunol (2004) 16(1):3-9. doi:10.1016/j.smim.2003.10.003

53. Demon D, Kuchmiy A, Fossoul A, Zhu Q, Kanneganti TD, Lamkanfi M. Caspase-11 is expressed in the colonic mucosa and protects against dextran sodium sulfate-induced colitis. Mucosal Immunol (2014) 7(6):1480-91. doi:10.1038/mi.2014.36

54. Kawashima T, Kosaka A, Yan H, Guo Z, Uchiyama R, Fukui R, et al. Doublestranded RNA of intestinal commensal but not pathogenic bacteria triggers production of protective interferon-beta. Immunity (2013) 38(6):1187-97. doi:10.1016/j.immuni.2013.02.024

55. Abt MC, Osborne LC, Monticelli LA, Doering TA, Alenghat T, Sonnenberg GF, et al. Commensal bacteria calibrate the activation threshold of innate antiviral immunity. Immunity (2012) 37(1):158-70. doi:10.1016/j.immuni. 2012.04.011

56. Reuter BK, Pizarro TT. Commentary: the role of the IL-18 system and other members of the IL-1R/TLR superfamily in innate mucosal immunity and the pathogenesis of inflammatory bowel disease: friend or foe? Eur J Immunol (2004) 34(9):2347-55. doi:10.1002/eji.200425351

57. Liu W, Guo W, Wu J, Luo Q, Tao F, Gu Y, et al. A novel benzo[d]imidazole derivate prevents the development of dextran sulfate sodium-induced murine experimental colitis via inhibition of NLRP3 inflammasome. Biochem Pharmacol (2013) 85(10):1504-12. doi:10.1016/j.bcp.2013.03.008

58. Wang Y, Wang H, Qian C, Tang J, Zhou W, Liu X, et al. 3-(2-Oxo-2phenylethylidene)-2,3,6,7-tetrahydro-1 $\mathrm{H}$-pyrazino[2,1-a] isoquinolin-4(1 1bH)-one (compound 1), a novel potent Nrf2/ARE inducer, protects against DSS-induced colitis via inhibiting NLRP3 inflammasome. Biochem Pharmacol (2016) 101:71-86. doi:10.1016/j.bcp.2015. 11.015

59. Dashdorj A, Jyothi KR, Lim S, Jo A, Nguyen MN, Ha J, et al. Mitochondriatargeted antioxidant MitoQ ameliorates experimental mouse colitis by suppressing NLRP3 inflammasome-mediated inflammatory cytokines. BMC Med (2013) 11:178. doi:10.1186/1741-7015-11-178

60. Masters SL, Dunne A, Subramanian SL, Hull RL, Tannahill GM, Sharp FA, et al. Activation of the NLRP3 inflammasome by islet amyloid polypeptide provides a mechanism for enhanced IL-1beta in type 2 diabetes. Nat Immunol (2010) 11(10):897-904. doi:10.1038/ni.1935

61. Wang X, Wang S, Hu C, Chen W, Shen Y, Wu X, et al. A new pharmacological effect of levornidazole: inhibition of NLRP3 inflammasome activation. Biochem Pharmacol (2015) 97(2):178-88. doi:10.1016/j.bcp.2015. 06.030

62. Liu X, Zhou W, Zhang X, Lu P, Du Q, Tao L, et al. Dimethyl fumarate ameliorates dextran sulfate sodium-induced murine experimental colitis by activating Nrf2 and suppressing NLRP3 inflammasome activation. Biochem Pharmacol (2016) 112:37-49. doi:10.1016/j.bcp.2016. 05.002

63. Itoh K, Tong KI, Yamamoto M. Molecular mechanism activating Nrf2-Keap1 pathway in regulation of adaptive response to electrophiles. Free Radic Biol Med (2004) 36(10):1208-13. doi:10.1016/j.freeradbiomed.2004.02.075
64. Guo W, Hu S, Elgehama A, Shao F, Ren R, Liu W, et al. Fumigaclavine C ameliorates dextran sulfate sodium-induced murine experimental colitis via NLRP3 inflammasome inhibition. J Pharmacol Sci (2015) 129(2):101-6. doi:10.1016/j.jphs.2015.05.003

65. Liu W, Guo W, Hang N, Yang Y, Wu X, Shen Y, et al. MALT1 inhibitors prevent the development of DSS-induced experimental colitis in mice via inhibiting NF-kappaB and NLRP3 inflammasome activation. Oncotarget (2016) 7(21):30536-49. doi:10.18632/oncotarget.8867

66. Seo SU, Kamada N, Muñoz-Planillo R, Kim YG, Kim D, Koizumi Y, et al. Distinct commensals induce interleukin-1beta via NLRP3 inflammasome in inflammatory monocytes to promote intestinal inflammation in response to injury. Immunity (2015) 42(4):744-55. doi:10.1016/j.immuni. 2015.03.004

67. Guo W, Liu W, Jin B, Geng J, Li J, Ding H, et al. Asiatic acid ameliorates dextran sulfate sodium-induced murine experimental colitis via suppressing mitochondria-mediated NLRP3 inflammasome activation. Int Immunopharmacol (2015) 24(2):232-8. doi:10.1016/j.intimp.2014.12.009

68. Wu XF, Ouyang ZJ, Feng LL, Chen G, Guo WJ, Shen Y, et al. Suppression of NF-kappaB signaling and NLRP3 inflammasome activation in macrophages is responsible for the amelioration of experimental murine colitis by the natural compound fraxinellone. Toxicol Appl Pharmacol (2014) 281(1):146-56. doi:10.1016/j.taap.2014.10.002

69. Sun Y, Zhao Y, Yao J, Zhao L, Wu Z, Wang Y, et al. Wogonoside protects against dextran sulfate sodium-induced experimental colitis in mice by inhibiting NF-kappaB and NLRP3 inflammasome activation. Biochem Pharmacol (2015) 94(2):142-54. doi:10.1016/j.bcp.2015.02.002

70. He X, Wei Z, Wang J, Kou J, Liu W, Fu Y, et al. Alpinetin attenuates inflammatory responses by suppressing TLR4 and NLRP3 signaling pathways in DSS-induced acute colitis. Sci Rep (2016) 6:28370. doi:10.1038/ srep 28370

71. Márquez-Flores YK, Villegas I, Cárdeno A, Rosillo MÁ, Alarcón-de-la-Lastra C. Apigenin supplementation protects the development of dextran sulfate sodium-induced murine experimental colitis by inhibiting canonical and non-canonical inflammasome signaling pathways. J Nutr Biochem (2016) 30:143-52. doi:10.1016/j.jnutbio.2015.12.002

72. Coll RC, Robertson AA, Chae JJ, Higgins SC, Muñoz-Planillo R, Inserra MC, et al. A small-molecule inhibitor of the NLRP3 inflammasome for the treatment of inflammatory diseases. Nat Med (2015) 21(3):248-55. doi:10.1038/ nm.3806

73. Dinarello CA, Simon A, van der Meer JW. Treating inflammation by blocking interleukin-1 in a broad spectrum of diseases. Nat Rev Drug Discov (2012) 11(8):633-52. doi:10.1038/nrd3800

74. Stoffels B, Hupa KJ, Snoek SA, van Bree S, Stein K, Schwandt T, et al. Postoperative ileus involves interleukin-1 receptor signaling in enteric glia. Gastroenterology (2014) 146(1):176.e-87.e. doi:10.1053/j.gastro.2013. 09.030

75. Coccia M, Harrison OJ, Schiering C, Asquith MJ, Becher B, Powrie F, et al. IL-1beta mediates chronic intestinal inflammation by promoting the accumulation of IL-17A secreting innate lymphoid cells and CD4(+) Th17 cells. J Exp Med (2012) 209(9):1595-609. doi:10.1084/jem. 20111453

76. Lissner D, Schumann M, Batra A, Kredel LI, Kühl AA, Erben U, et al. Monocyte and M1 macrophage-induced barrier defect contributes to chronic intestinal inflammation in IBD. Inflamm Bowel Dis (2015) 21(6):1297-305. doi:10.1097/MIB.0000000000000384

Conflict of Interest Statement: The authors declare that the research was conducted in the absence of any commercial or financial relationships that could be construed as a potential conflict of interest.

Copyright (c) 2017 Pellegrini, Antonioli, Lopez-Castejon, Blandizzi and Fornai. This is an open-access article distributed under the terms of the Creative Commons Attribution License (CC BY). The use, distribution or reproduction in other forums is permitted, provided the original author(s) or licensor are credited and that the original publication in this journal is cited, in accordance with accepted academic practice. No use, distribution or reproduction is permitted which does not comply with these terms. 